\title{
Phase transition of the nucleon-antinucleon plasma in a relativistic mean-field theory
}

\author{
J. Theis \\ Gesellschaft für Schwerionenforschung, Darmstadt, West Germany \\ G. Graebner, G. Buchwald, J. Maruhn, and W. Greiner \\ Institut für Theoretische Physik, Universität Frankfurt, Frankfurt, West Germany \\ H. Stöcker \\ Department of Physics and Astronomy and \\ National Superconducting Cyclotron Laboratory, Michigan State University, East Lansing, Michigan 48824 \\ J. Polonyi \\ Department of Physics, University of Illinois, Urbana, Illinois 61801
}

(Received 27 May 1983)

\begin{abstract}
Studying Walecka's mean-field theory we find that one can reproduce the observed binding ener"gy and density of nuclear matter within experimental precision in an area characterized by a line in the coupling-constant plane. A part of this line defines systems which exhibit a phase transition around $T_{c} \sim 200 \mathrm{MeV}$ for zero baryon density. The rest corresponds to such systems where the phase transition is absent; in that case a peak appears in the specific heat around $T \sim 200 \mathrm{MeV}$. We interpret these results as indicating that the hadron phase of nuclear matter alone indicates the occurrence of an abrupt change in the bulk properties around $\rho_{V} \sim 0$ and $T \sim 200 \mathrm{MeV}$.
\end{abstract}

\section{INTRODUCTION}

A long-standing aim of theoretical nuclear physics is to describe the bulk properties of hadronic matter. An ambitious hope is that this task can be accomplished at least numerically by doing lattice QCD calculations in the near future. Even if we had such results to date, it is still very instructive to study effective theories describing hadronic matter in a definite range of temperature and density. One of the most successful effective models is Walecka's mean-field theory. ${ }^{1-5}$ On the one hand, it describes suprisingly well a variety of physical properties of nuclear matter and of finite nuclei, and on the other hand it can be generalized to contain the linear $\sigma$ model which is supposed to be the low-energy effective theory of strong interactions. ${ }^{6}$ In the following we extrapolate the effective theory to the high-temperature region $(T>200 \mathrm{MeV})$. This extrapolation may be somewhat misleading, since scalar and vector mesons are not sufficient for nuclear interaction at this temperature. Nevertheless it is of value to study the nonlinear behavior of such an effective theory under extreme conditions.

We expect that a phase transition takes place in hadronic matter at high temperature or density. Having an effective theory in the low-temperature or -density region, one can study whether this model contains a phase transition. This procedure can be contrasted with another strategy, according to which two different approximations are used (e.g., Walecka's theory and perturbative QCD in the case of quark matter) and one tries to match them.

In this article, we study Walecka's mean-field theory with the following results: (i) We can reproduce the observed binding energy and density of nuclear matter within experimental precision in an area characterized by a line in the coupling-constant plane. The couplingconstant values proposed by Walecka represent a point close to the end of this line. (ii) We have found that a part of this line defines systems which exhibit a phase transition around $T_{c} \sim 200 \mathrm{MeV}$ for zero baryon density. The rest of this line corresponds to such systems where the phase transition is absent but there is a peak in the specific heat around $T \sim 200 \mathrm{MeV}$. We interpret these results as indicating that the hadron phase of nuclear matter alone indicates the occurrence of an abrupt change in the bulk properties around $\rho_{V} \sim 0$ and $T \sim 200 \mathrm{MeV}$.

\section{DETERMINATION OF COUPLING CONSTANTS}

Walecka's nuclear field theory contains the baryon field $\psi$ and scalar- and vector-meson fields $\Phi$ and $V_{\mu}$, respectively. ${ }^{1,2}$ The field Lagrangian is given by

$$
\begin{aligned}
\mathscr{L}= & \int d r\left[-\psi\left(\gamma^{\mu} \partial_{\mu}+M\right) \psi\right]-\frac{1}{2}\left[\left(\partial^{v} \Phi\right)\left(\partial_{\nu} \Phi\right)+m_{S}{ }^{2} \Phi^{2}\right] \\
& -\frac{1}{2}\left(\frac{1}{2} F_{\lambda \rho} F^{\lambda \rho}+m_{V}{ }^{2} V_{\lambda} V^{\lambda}\right)+i g_{V} \psi \gamma^{\mu} \psi V_{\mu}+g_{S} \psi \psi \Phi,
\end{aligned}
$$

where $M, M_{S}$, and $m_{V}$ are the inverse Compton wavelengths of the baryon, scalar meson, and vector meson, respectively. Here the field tensor is $F_{\rho \lambda}=\partial_{\rho} V_{\lambda}-\partial_{\lambda} V_{\rho}$. The equations of motion obtained from Eq. (1) are

$$
\begin{aligned}
& {\left[\gamma^{\mu} \partial_{\mu}+\left(M-g_{S} \Phi\right)-i g_{V} \gamma^{\mu} V_{\mu}\right] \psi=0,} \\
& \left(-\partial_{t}{ }^{2}+\nabla^{2}-m_{S}{ }^{2}\right) \Phi=-g_{S} \rho_{s}, \\
& \left(-\partial_{t}{ }^{2}+\nabla^{2}-m_{V}{ }^{2}\right) V_{\mu}=-i g_{V} j_{\mu},
\end{aligned}
$$

with the scalar density $\rho_{S}=\bar{\psi} \psi$, the baryon (vector) density $\rho_{V}=j_{0}=\bar{\psi} \gamma_{0} \psi$, and the baryon current $\overrightarrow{\mathrm{j}}=\bar{\psi} \vec{\gamma} \psi$. Con-

(C) 1983 The American Physical Society 
sidering a uniform system of baryons the meson fields can be replaced by the classical fields. ${ }^{1,2}$ In this limit Eqs. (2) -(4) reduce to mean-field equations

$$
\begin{aligned}
& {\left[\gamma^{\mu} \partial_{\mu}+\left(M-g_{S} \bar{\Phi}\right)+g_{V} \gamma_{0} \bar{V}_{0}\right] \psi=0,} \\
& \bar{\Phi}=\left(g_{S} / m_{S}^{2}\right) \rho_{S}, \\
& \bar{V}_{0}=\left(g_{V} / m_{V}{ }^{2}\right) \rho_{V} .
\end{aligned}
$$

One can calculate thermodynamical quantities in the mean-field approximation and find ${ }^{3}$

$$
\begin{aligned}
e= & \frac{1}{2}\left(1 / C_{S}^{2}\right)(1-x)^{2}+\frac{1}{2} C_{V}^{2} n_{V}^{2} \\
& +\left[\gamma /(2 \pi)^{3}\right] \int d^{3} k\left(k^{2}+x^{2}\right)^{1 / 2}[n(\theta)+\bar{n}(\theta)],
\end{aligned}
$$

$$
\begin{aligned}
& p=-\frac{1}{2}\left(1 / C_{S}{ }^{2}\right)(1-x)^{2}+\frac{1}{2} C_{V}{ }^{2} n_{V}{ }^{2}+\frac{1}{3}\left[\gamma /(2 \pi)^{3}\right] \\
& \times \int d^{3} k k^{2}[n(\theta)+\bar{n}(\theta)] /\left(k^{2}+x^{2}\right)^{1 / 2}, \\
& n_{V}=\left[\gamma /(2 \pi)^{3}\right] \int d^{3} k[n(\theta)-\bar{n}(\theta)],
\end{aligned}
$$

where $e, p$, and $n_{V}$ are the dimensionless energy density, total pressure, and vector (baryon) density. The first two terms in Eqs. (8) and (9) arise from the scalar- and vector-meson fields, while the final two contributions are those of a relativistic Fermi gas of baryons with an effective mass $M^{*}=x M . \gamma$ is the degeneracy factor $(\gamma=4$ for nuclear matter), $n(\theta)$ and $\bar{n}(\theta)$ stand for the Fermi function for baryons and antibaryons, respectively. $\theta$ defines the dimensionless temperature $\theta=T / M c^{2}$. The nucleon effective mass $M^{*}=x M$ is given by the equation of motion of the scalar-meson field $\Phi$ :

$$
x=\left\{1+C_{S}^{2}\left[\gamma /(2 \pi)^{3}\right] \int d^{3} k[n(\theta)+\bar{n}(\theta)] /\left(k^{2}+x^{2}\right)^{1 / 2}\right\}^{-1} .
$$

In the original publication, ${ }^{1}$ the coupling constants $C_{S}{ }^{2}$, $C_{V}{ }^{2}$, were fixed to give $\epsilon_{\text {bind }}=-15.75 \mathrm{MeV}$ and $k_{F}=1.42$ $\mathrm{fm}^{-1}$. However, the resulting equilibrium density $\rho_{\text {eq }}=0.19 \mathrm{fm}^{-3}$ and the nuclear incompressibility $\kappa \sim 550$ $\mathrm{MeV}$ were unrealistically large. We have tried to fix these shortcomings by using different sets of coupling constants. Figure 1 shows the region of the coupling-constant plane where the mean-field solution yields the nuclear binding energy $-16<\epsilon_{\text {bind }}<-15 \mathrm{MeV}$ at equilibrium densities $0.14<\rho_{\text {eq }}<0.19 \mathrm{fm}^{-3}$. However, we observe that for any reasonable set of coupling constants the compression constant $\kappa$ does not change significantly, even for the lowest $\rho_{\text {eq }}$ values. Also the effective baryon mass $x=M^{*} / M$, which is the governing quantity of the meanfield approximation, is constant in this region within $1 \%$.

The physical reason for this wide latitude in the deter-

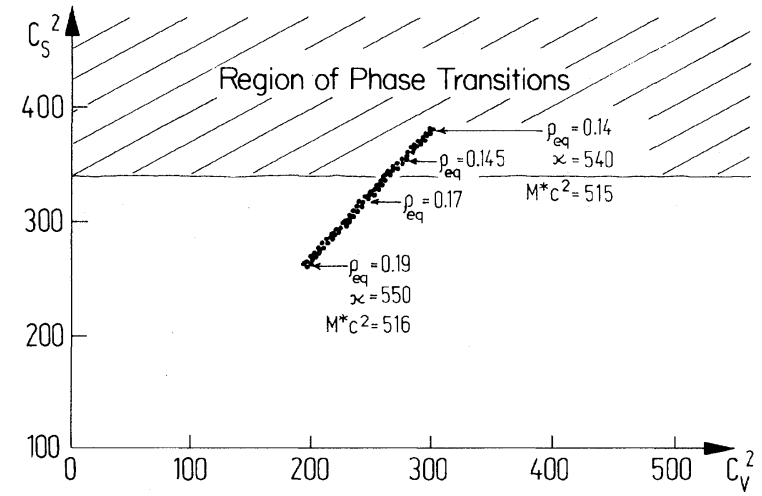

FIG. 1. The region of the coupling-constant plane where the mean-field solution reproduces the nuclear binding energy $-16<\epsilon<-15 \mathrm{MeV}$ at equilibrium densities $0.14<\rho_{\mathrm{eq}}<0.19$ $\mathrm{fm}^{-3}$. We observe that for any reasonable set of coupling constants the compression modulus $\kappa$ does not change significantly. Also the effective baryon mass $x=M^{*} / M$ is constant in this region up to $1 \%$. mination of the coupling constant is not completely clear. Mathematically the situation is the following: By fixing the binding energy one gets a line in the coupling-constant plane. Moving along this line the scalar coupling constant $C_{S}{ }^{2}$ and the Fermi momentum $k_{F}$ corresponding to minimal energy (at $\theta=0$ ) change. These changes are related such that they almost cancel each other in the second term of the denominator in the self-consistency equation (11). In this way the effective mass changes very slowly along the line and one has an elongated region in the plane of coupling constants giving approximately the same $\epsilon_{\text {bind }}$ and $k_{F}$.

\section{PHASE STRUCTURE}

Let us now explore the phase structure for the effective Lagrangian at vanishing chemical potential $\mu$ and vector density $\rho_{v}\left(\mu=\rho_{V}=0\right)$. First observe that for $\mu=0$ the vector coupling constant does not appear in the thermodynamical quantities (8)-(11) calculated in the mean-field approximation. It is interesting that this feature is preserved by the leading-order quantum corrections as well. ${ }^{4,7}$

In contrast to $\mu \neq 0$, the Fermi integration can be done analytically, yielding for the dimensionless energy density

$$
e=\frac{1}{2}\left(1 / C_{S}{ }^{2}\right)(1-x)^{2}+U
$$

with the internal energy density

$$
\begin{aligned}
& U=\left(\gamma \theta x^{3} / \pi^{2}\right) \sum\left[(-1)^{n-1} / n\right][ K_{1}(n x / \theta) \\
&\left.+(3 \theta / n x) K_{2}(n x / \theta)\right]
\end{aligned}
$$

and the total pressure

$$
p=-\frac{1}{2}\left(1 / C_{S}{ }^{2}\right)(1-x)^{2}+p_{b},
$$

with the baryonic contribution 


$$
p_{b}=\left(\gamma \theta^{2} x^{2} / \pi^{2}\right) \sum\left[(-1)^{n-1} / n^{2}\right] K_{2}(n x / \theta) .
$$

The effective mass $M^{*}=x M$ is defined by the selfconsistency relation

$$
x=\left\{1+\left(\gamma C_{S}^{2} \theta x / \pi^{2}\right) \sum\left[(-1)^{n-1} / n\right] K_{1}(n x / \theta)\right\}^{-1} .
$$

Here $K_{1}$ and $K_{2}$ stand for the modified Bessel functions of first and second order, respectively.

Figure 2 contains the solution $x$ of the self-consistency equation (16) as a function of the temperature. The general trend of the function $x(\theta)$ can be understood as follows: The scalar density, which appears as a source for the scalar-meson field $\Phi$, increases with increasing temperature. This leads to an increase of the mean-field value of $\Phi$. Since the scalar meson describes an attractive interaction of the nucleons, these will be bound more strong$1 y$, and thus the effective mass is reduced. This mechanism is reinforced by the fact that a decrease of the effective mass increases the scalar density again.

The most striking feature is the sudden drop in $x$ at temperature $T \sim 200 \mathrm{MeV}$ where the transition from $x=0.75$ to $x=0.25$ occurs in an interval of $\Delta T \sim 5-10$ MeV only.
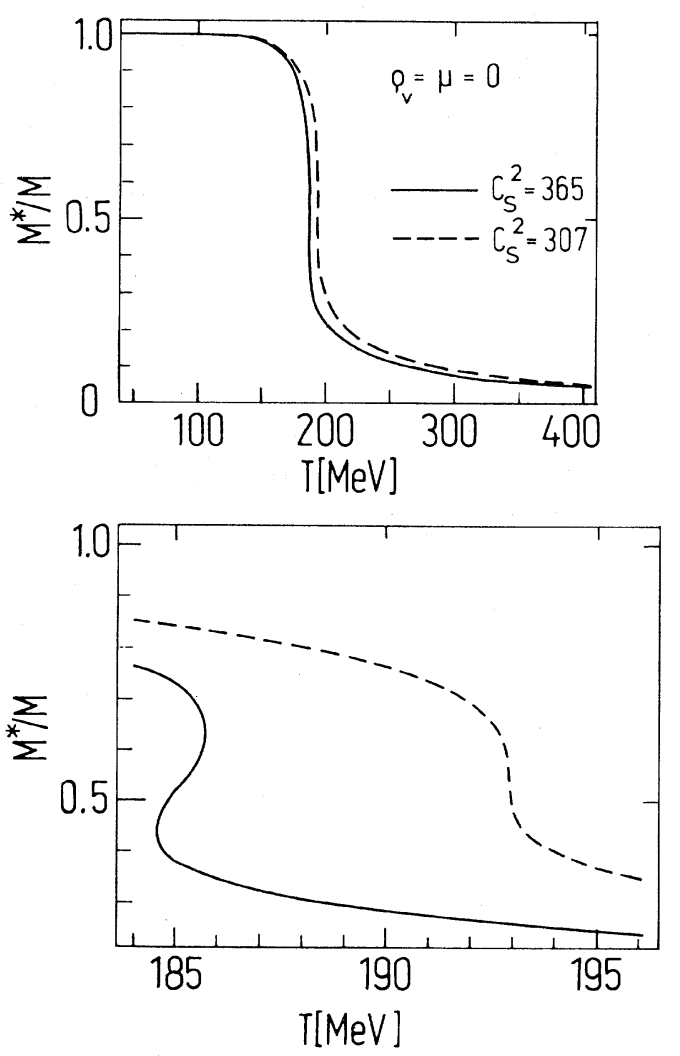

FIG. 2. For $\rho_{v}=\mu=0$ the solution $x=M^{*} / M$ of the selfconsistency equation (16) is plotted as a function of temperature for different values of $C_{S}{ }^{2}$. The lower graph contains the same results but with a stretched temperature axis near the transition point.
We have computed thermodynamical quantities for the various coupling constants of the regions shown in Fig. 1. For $C_{S}{ }^{2}>342$ we find a phase transition of first order. For $C_{S}{ }^{2}<342$ the thermodynamical behavior is smooth in the temperature but a peak of finite width is found in the specific heat (see Fig. 5). For $C_{S}{ }^{2}=342$ there is a phase transition of second order.

To the best of our knowledge this is the first example in the literature of an effective theory in which the order of the phase transition directly depends on the strength of the coupling constant.

Figures 3-5 show the temperature dependence of the total energy density divided by the high-temperature limit of the energy density [Eq. (12)], the pressure, and the
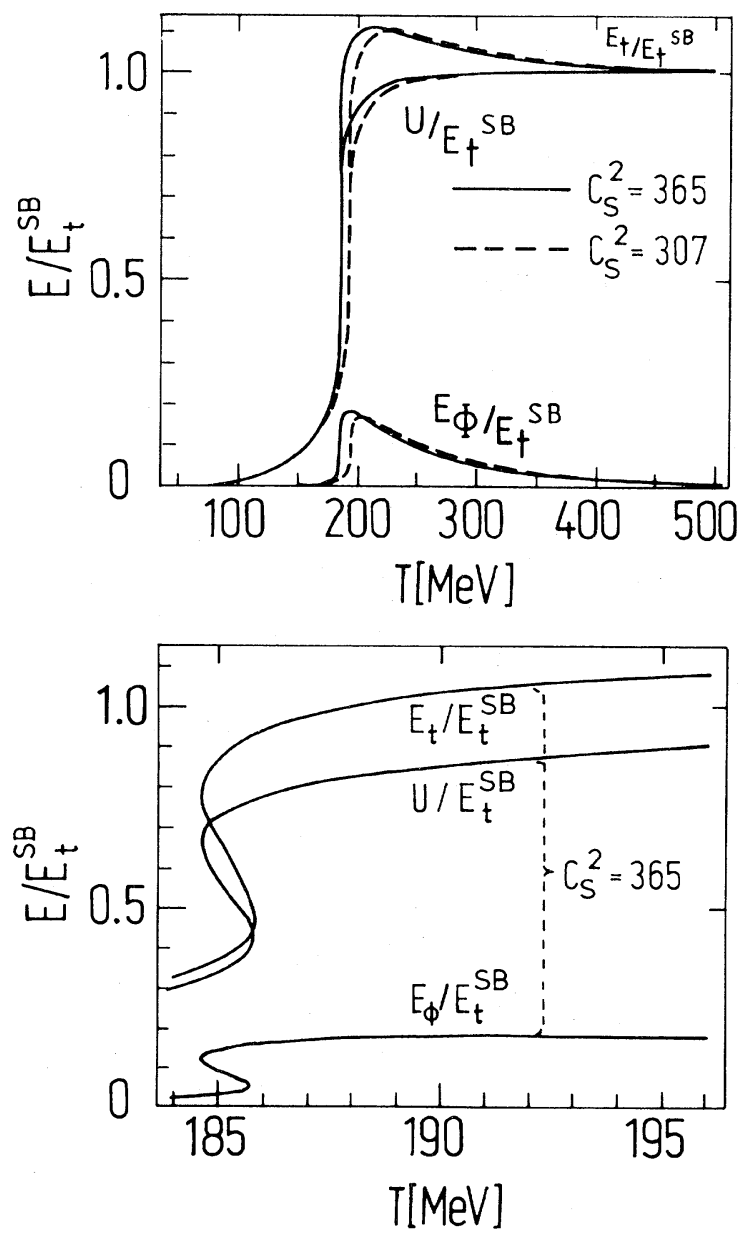

FIG. 3. For $\rho_{v}=\mu=0$ the temperature dependence of the normalized energy densities is shown. $E_{t}, U$, and $E_{\Phi}$ stand for the total, nuclear, and scalar-field energy densities, respectively. They are divided by the Stefan-Boltzmann limit of $E_{t}^{\mathrm{SB}}$. From $E_{t}^{\mathrm{SB}}$ one recognizes that the system reaches its high-temperature behavior quite abruptly. Since the effective mass is very small at high temperature the system suddenly decouples to almost free zero mass nucleons, i.e., up to the constant first term in the energy density and pressure. How this happens depends on the actual values of the coupling constants $C_{S}{ }^{2}$. The lower graph contains the results only for $C_{S}{ }^{2}=365$ but with a stretched temperature axis. 

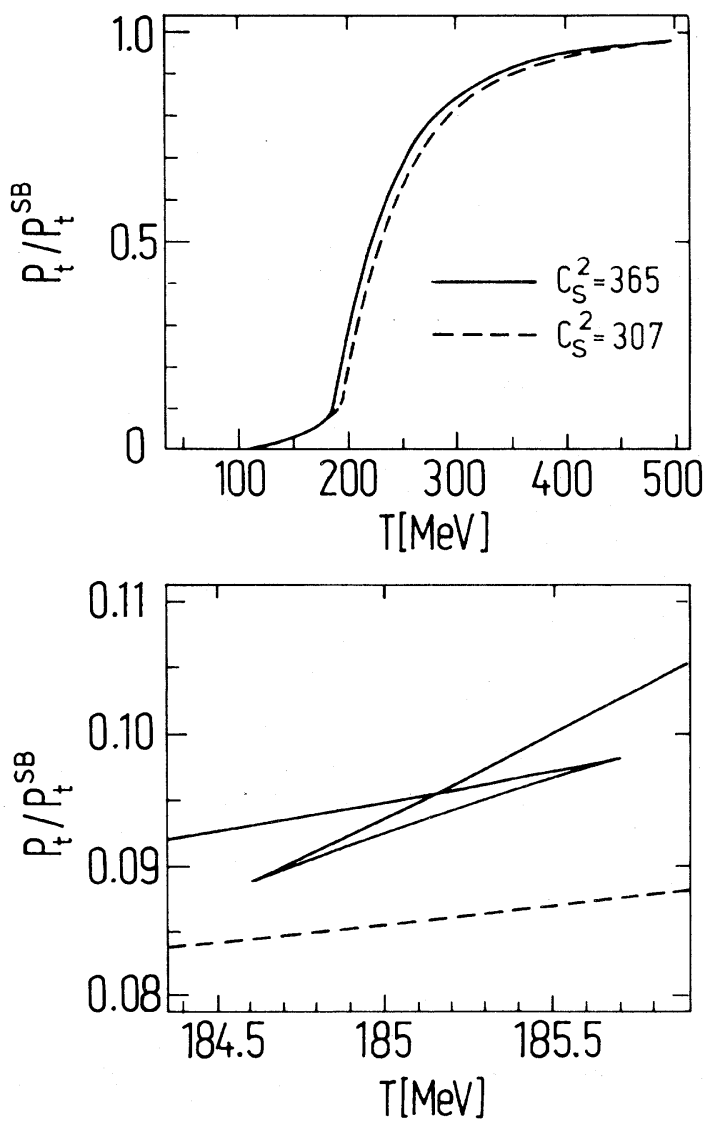

FIG. 4. For $\rho_{v}=\mu=0$ total pressure divided by the hightemperature (Stefan-Boltzmann) limit is plotted versus temperature for different coupling constants $C_{S}{ }^{2}$. The lower graph shows the transition point.

specific heat for $C_{S}{ }^{2}=307\left(\rho_{\mathrm{eq}}=0.17\right)$ and $C_{S}{ }^{2}=365$ $\left(\rho_{\text {eq }}=0.145\right)$. The total energy density shows (Fig. 3) that the system reaches its high-temperature behavior quite abruptly around $T \sim 200 \mathrm{MeV}$. Since the effective mass is very small at high temperature, we can say that the system suddenly decouples to almost free zero-mass nucleons, i.e., up to the constant first term in the energy density and pressure. How this happens depends on the actual values of the coupling constants $C_{S}{ }^{2}$. It must be kept in mind, though, that this picture comes from the mean-field approximation so that the detailed structure of this sudden change (e.g., whether we observe a phase transition or a peak of finite width in the specific energy only) may be different in the full quantum field theory.

Another remark concerns the observation that at high temperature the system behaves like an almost-free zeromass fermion gas with a constant shift in the energy density and in the pressure. This is quite analogous to the expected chiral phase transition in high-temperature QCD. ${ }^{8}$ However we should keep in mind that there is no liberation of internal degrees of freedom of hadrons in the Walecka model, i.e., the phase transition found here can-
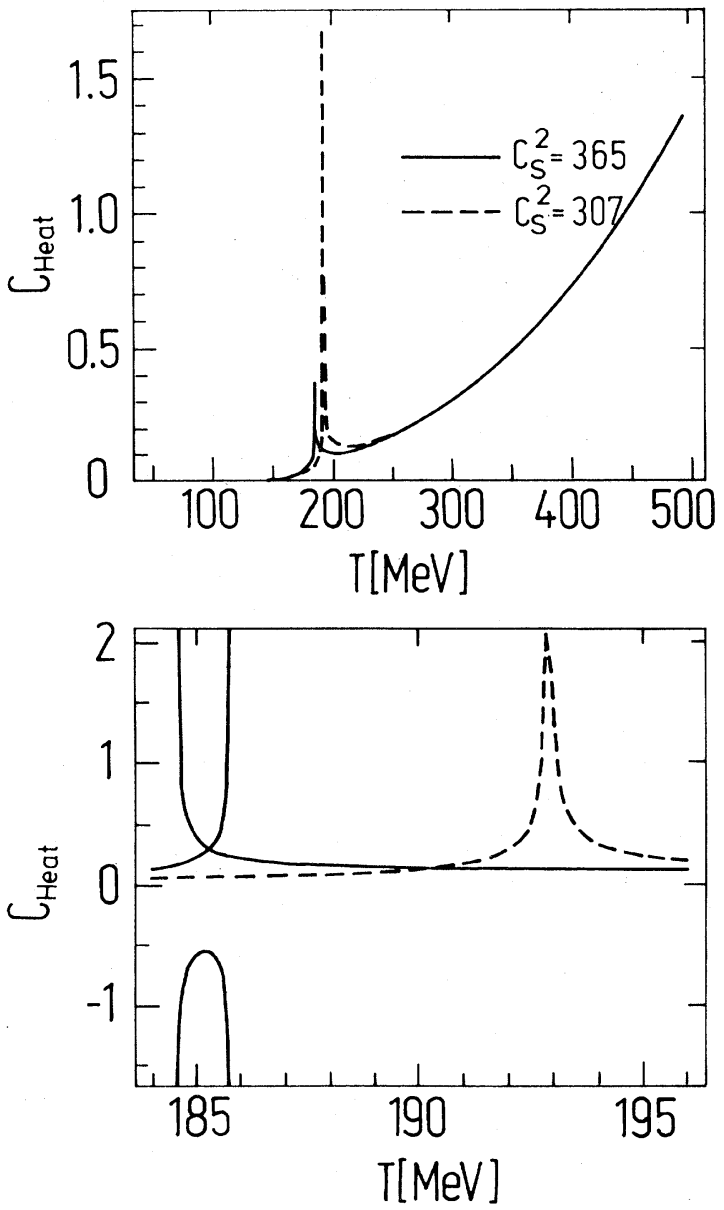

FIG. 5. The dimensionless specific heat $C_{\text {Heat }}$ is shown as a function of temperature and for different $C_{S}{ }^{2}$. Because of the linearity of $C_{\text {Heat }}$ in $d x / d \theta$ and the pole structure of $d x / d \theta$, the specific heat diverges for $C_{S}{ }^{2}=365$, but is continuous for $C_{S}{ }^{2}=307$.

not be interpreted as a transition from baryon to quark matter. ${ }^{9}$

\section{CRITICAL LINE OF THE MEAN-FIELD THEORY}

The mathematical structure of the self-consistency equation is so simple that it is possible to understand how this decoupling happens. First we remark that the specific heat calculated from (12) is linear in $d x / d \theta$. So whenever we see a sudden fall in $x(\theta)$, there is a peak in the specific heat (see Fig. 5). In addition, if we have three solutions of the self-consistency equation, similarly the total energy density in that region is also triple valued. This type of temperature dependence leads to a phase transition of first order applying the Maxwell construction in order to avoid instability. In this way the fact that we see one or two poles or only a peak in $d x / d \theta$ means, respectively, that we have a phase transition of second or first order or continuous thermodynamical behavior.

The next step is to clarify the pole structure of $d x / d \theta$. It is easy to express $d x / d \theta$ as a function of $x$ and $\theta$ : 


$$
d x / d \theta=\frac{\left(-\gamma C_{S}{ }^{2} x^{3} / \pi^{2} \theta\right) \sum\left[(-1)^{n-1} / n\right] K_{2}}{1+\left(\gamma C_{S}{ }^{2} x \theta / \pi^{2}\right) \sum\left[(-1)^{n-1} / n\right]\left[K_{1}-(n x / \theta) K_{0}\right]} .
$$

The poles of this expression in the $(x, \theta)$ plane lie on the curve determined by the vanishing of the denominator. Using Eq. (16) this condition becomes

$$
0=1-\left(\gamma C_{S}^{2} x^{3} / \pi^{2}\right) \sum\left[(-1)^{n-1}\right] K_{0} .
$$

The number of intersections of this line with the solution of the self-consistency equation tells us how the decoupling happens. The numerical study of this question leads to the following results:

(i) For $C_{S}{ }^{2}<342$ the lines do not cross, so that for such coupling constants the decoupling is continuous.

(ii) For $C_{S}{ }^{2} \simeq 342$ the lines have one point of contact so that we have a phase transition of second order at $T_{c}=205 \mathrm{MeV}$ where $x=0.505$ (these values come from the $x, T$ coordinates of the point of contact).

(iii) For $C_{S}^{2}>342$ the lines cross twice, so in this case we have a phase transition of first order. The critical temperature and $x$ at both end points of the mixed phase are given by the $x, T$ coordinates of the intersections.

Thus the order of the phase transition strongly depends on the actual value of the coupling constant $C_{S}{ }^{2}$.

\section{SUMMARY}

We have studied the equation of state of the baryonantibaryon plasma at high temperature and at zero baryon density. Our result is that around temperature $T \sim 200$

$\mathrm{MeV}$ the pressure and the internal energy become, up to a constant, those of a free-massless-fermion gas having the degeneracy factor of nuclear matter. This sudden change can be manifested as a peak in the specific heat or as a phase transition depending on the actual values of coupling constants. In addition, we have found that if one fixes the coupling constants by fitting the measured binding energy and density for nuclear matter to experimental values, the possible coupling-constant values determine an elongated strip in the coupling-constant plane. This strip contains coupling-constant values corresponding to both types of the mentioned thermodynamical behavior. We interpret these results such that the nuclear field theory, as a low-temperature effective theory of hadronic matter, indicates the occurrence of a sudden change in thermodynamical behavior around $T_{c} \sim 200 \mathrm{MeV}$ at zero baryon density, in analogy to quark deconfinement in lattice QCD calculations.

\section{ACKNOWLEDGMENT}

The authors would like to thank Professor Dr. B. Müller for constructive discussions and his interest in this work.
1J. D. Walecka, Ann. Phys. (N.Y.) $\underline{83}, 491$ (1974).

${ }^{2}$ S. A. Chin and J. D. Walecka, Phys. Lett. 52B, 24 (1974).

3J. D. Walecka, Phys. Lett. 59B, 109 (1975).

${ }^{4}$ R. A. Freedman, Phys. Lett. $\underline{71 B}, 369$ (1977).

${ }^{5} \mathrm{~S}$. Garpmann, N. Glendenning, and Y. Karant, Nucl. Phys. A322, 382 (1979). These authors included nucleon resonances into the mean-field theory. The resonances, as well as the thermal gases of scalar and vector mesons are not included in this work.

6J. Boguta and A. Bodmer, Nucl. Phys. A292, 413 (1977); J. Boguta and H. Stöcker, Phys. Lett. 120B, 289 (1983).

${ }^{7}$ S. A. Chin, Phys. Lett. 62B, 263 (1976).
${ }^{8}$ I. Montvay and E. Pietarinen, Phys. Lett. 115B, 151 (1982).

${ }^{9}$ This effective theory may, however, be useful in studying the influence of such a first-order phase transition on the reaction dynamics in complex, three-dimensional dynamical calculations, as opposed to the simplified models used up to date to describe the hadron-quark transition in nuclear collisions: see, for example, S. A. Chin, Phys. Lett. 78B, 552 (1978); K. A.

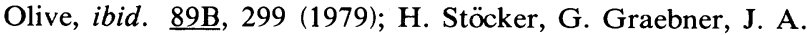
Maruhn, and W. Greiner, ibid. 95B, 192 (1980); Z. Phys. A 295, 40 (1980); R. Anishetty, P. Koehler, and L. McLerran, Phys. Rev. D 22, 2793 (1980); G. Domokos and J. I. Goldman, ibid. 23, (1981). 